\title{
Recent Results from Analysis of Flow Structures and Energy Modes Induced by Viscous Wave around a Surface-Piercing Cylinder
}

\author{
Giancarlo Alfonsi, Agostino Lauria, and Leonardo Primavera \\ Fluid Dynamics Laboratory, Università della Calabria, Via P. Bucci 42b, Rende, 87036 Cosenza, Italy \\ Correspondence should be addressed to Giancarlo Alfonsi; giancarlo.alfonsi@unical.it
}

Received 16 March 2017; Revised 2 July 2017; Accepted 2 October 2017; Published 12 November 2017

Academic Editor: Sandro Longo

Copyright (C) 2017 Giancarlo Alfonsi et al. This is an open access article distributed under the Creative Commons Attribution License, which permits unrestricted use, distribution, and reproduction in any medium, provided the original work is properly cited.

\begin{abstract}
Due to its relevance in ocean engineering, the subject of the flow field generated by water waves around a vertical circular cylinder piercing the free surface has recently started to be considered by several research groups. In particular, we studied this problem starting from the velocity-potential framework, then the implementation of the numerical solution of the Euler equations in their velocity-pressure formulation, and finally the performance of the integration of the Navier-Stokes equations in primitive variables. We also developed and applied methods of extraction of the flow coherent structures and most energetic modes. In this work, we present some new results of our research directed, in particular, toward the clarification of the main nonintuitive character of the phenomenon of interaction between a wave and a surface-piercing cylinder, namely, the fact that the wave exerts its maximum force and exhibits its maximum run-up on the cylindrical obstacle at different instants. The understanding of this phenomenon becomes of crucial importance in the perspective of governing the entity of the wave run-up on the obstacle by means of wave-flow-control techniques.
\end{abstract}

\section{Introduction}

Wave diffraction caused by vertical circular cylinders piercing the free surface has gained a renewed relevance in ocean engineering due to recent technical applications such as the installation of offshore wind turbines, where, in the case of small amplitude waves, the results of numerical and experimental studies are usually compared with the potential function solution introduced by the pioneering work of MacCamy and Fuchs [1]. As for published works, in the numerical field, there exist a number of potential-flow studies [2-5] in which the good correspondence between potential flow fields and potential theory results is clearly demonstrated. In the experimental field, on the contrary, a number of authors [6-20] verified that linear diffraction actually strongly underestimates the run-ups (for a more extensive discussion about existing literature, one can refer to the review work [21]). In fact, under a computational viewpoint, the only correct approach to this problem is the integration of the system of the Navier-Stokes equations, a procedure still rarely used today. One of the first researches of this kind is that of Choi et al. [22] who executed a study of the run-up induced by a tsunami wave around a simplified island model adopting the RANS technique coupled with an RNG closure model (see [23] for an overview of the RANS approach to the numerical integration of the Navier-Stokes equations, and refer to Nomenclature for the meaning of acronyms and symbols). Alfonsi et al. [24-26] have faced the problem of wave diffraction caused by a circular cylinder piercing the free surface, starting from the velocity-potential approach, then the solution of the Euler equations in their velocity-pressure formulation, and finally, numerically solving the system of the Navier-Stokes equations in primitive variables, following the DNS technique. Methods for the extraction of the flow coherent structures ([27]) and most energetic modes have also been developed and applied. In this work some new results of our research are presented, in particular, directed toward the explanation of the main nonintuitive character of the phenomenon of 
interaction between a water wave and a surface-piercing vertical circular cylinder, namely, the fact that the wave exerts its maximum force and exhibits its maximum runup on the cylindrical obstacle at different instants. In what follows, Section 2 reports on numerical simulations and code validation and Section 3 concisely reports on the methods followed for the extraction of the flow coherent structures and most energetic modes, while, in Section 4, the results are illustrated. A discussion is reported in the conclusions.

\section{Calculations}

The nondimensional Navier-Stokes equations, in three dimensions and in their velocity-pressure formulation, are considered (the fluid is incompressible and viscous, Einstein summation convention applies to repeated indices, $i, j=$ $1,2,3)$ :

$$
\begin{aligned}
\frac{\partial u_{i}}{\partial t}+u_{j} \frac{\partial u_{i}}{\partial x_{j}} & =-\frac{\partial p}{\partial x_{i}}+\frac{1}{\operatorname{Re}_{\mathrm{NS}}} \frac{\partial^{2} u_{i}}{\partial x_{j} \partial x_{j}}+\delta_{i, 3} \\
\frac{\partial u_{i}}{\partial x_{i}} & =0
\end{aligned}
$$

where $u_{i}(u, v, w)$ are nondimensional velocity components in the Cartesian-coordinate system $x_{i}(x, y, z), p$ is a nondimensional pressure, and $\delta_{i j}$ is the Kronecker delta (note that, for simplicity, the symbols for both dependent and independent variables have not been altered in switching from the dimensional to the dimensionless formalism). Operators and variables have been adimensionalized by the cylinder diameter $(D)$ for lengths, the groups $(\sqrt{D g})$ for velocities, $(\rho D g)$ for pressures, and $(\sqrt{D / g})$ for time, and $\operatorname{Re}_{\mathrm{NS}}$ is the Reynolds number that results from the above adimensionalization operations ( $\rho$ is fluid density, $\nu$ is fluid kinematic viscosity, $g$ is acceleration due to gravity).

The Reynolds number $\left(\mathrm{Re}_{\mathrm{NS}}\right)$ in equation (1a) and the wave-field Reynolds number (Re), are related by the following expression:

$$
\operatorname{Re}_{\mathrm{NS}}=\frac{\sqrt{D g} D}{v}=\frac{\sqrt{2 \pi} \operatorname{Re}}{\mathrm{KC} \sqrt{\frac{D}{L} \tanh (k d)}}
$$

The Flow-3D code has been utilized in the computations $[28,29]$. This computational code solves the flow momentum equations using the finite-volumes, and has been satisfactorily validated by several authors in different flow cases [3034].

We have also performed a work of validation of the code. The Flow-3D solver has been validated in the case of the flow of a viscous fluid between two parallel plates at a moderate Reynolds-number value, against an accurate reference code, that is, the hybrid spectral-finite difference Navier-Stokes code, as originally developed by Alfonsi et al. [35], and later tested by Passoni et al. [36-38], and by Alfonsi and Primavera [39] (one can refer to Table 3 of [21] for an outlook of the computational parameters used in the numerical simulations of the benchmark case). The process of code validation gave very satisfactory results, as related to both mean-flow quantities and high-order statistics of fluctuating velocities. Thus, we are now confident that high-energy modes and coherent structures of the flow, as computed in the present work, are correct (one can refer to Figures 4-8 of [21] for an extensive presentation of the benchmark-case results).

In the present simulations, no models have been used for the fluctuating velocities, so we follow the technique of the Direct Numerical Simulation (DNS). It is known that in DNS one solves the system of the momentum equations with physically-consistent accuracy in both time and space, that is, one resolves the essential spatial and temporal scales of the flow. Concerning the scale-resolution requirements in DNS, the criterion of resolving the Kolmogorov microscales $\left[\eta=\left(\nu^{3} / \bar{\varepsilon}\right)^{1 / 4}, \tau_{\eta}=(\nu / \bar{\varepsilon})^{1 / 2}\right]$ is usually followed, or at most limited multiples of the latter. The Kolmogorov microscales have been calculated by estimating the quantity $(\bar{\varepsilon})$, that is, the average rate of dissipation of kinetic energy per unit mass [40]:

$$
\bar{\varepsilon} \cong \frac{2 \pi r_{0} d \tau_{w} u_{\max }}{\rho\left(L_{x} L_{y} d-\pi r_{0}^{2} d\right)}=\frac{F_{f} u_{\max }}{\rho d\left(L_{x} L_{y}-\pi r_{0}^{2}\right)}
$$

( $F_{f}$ is the portion of the force due to friction [41]), so that one obtains

$$
\begin{aligned}
\bar{\varepsilon} & \cong 0.00000525 D^{1 / 2} g^{3 / 2} \\
\eta & =0.0147 D \\
\tau_{\eta} & =3.45 \sqrt{\frac{D}{g}}
\end{aligned}
$$

The computational domain ([21], Figure 3) has been discretized in a 5-block computational grid ([21], Figure 9).

Table 4 of [21] reports (besides other relevant parameters) the partitions of the computing domain occupied by each block of grid points, and the grid-point distances $\Delta x, \Delta y, \Delta z$ within each block. As for the computational grid, it can be noticed that (i) the grid point-distances $\Delta x$ and $\Delta y$ (along the $x$ - and $y$-directions) are constant in the peripheral blocks $1,2,4$, and 5, while, within the internal block 3 , they are nonuniformly distributed according to a hyperbolic-tangent law, (ii) $\Delta z$ (along the $z$-direction) assumes a constant value through all blocks. By adopting this configuration, the space resolutions along $x$ and $y$ at the cylinder wall can resolve less than $0.5 \eta$, while at the outer boundaries of the computing domain they can still resolve less than $2.5 \eta$ (one can also refer to [21], Table 4). These values are well in the ranges usually used in DNS. No-slip and zero wall-normal velocity boundary conditions have been imposed on the cylinder wall. Symmetry conditions have been set on both the $x-z$ lateral boundary planes of the domain, outflow conditions have been enforced on the $y-z$ end-plane, besides the free-surface condition at the wave surface. The flow field corresponding to the incident wave of the case at hand has been generated at the $y-z$ inlet plane. A specially-assembled multicore computer has been used for the calculations; it includes 2 Intel Xeon 5660 exa-core processors (a total of $12 \mathrm{CPU}$ ), $48 \mathrm{~GB}$ of 

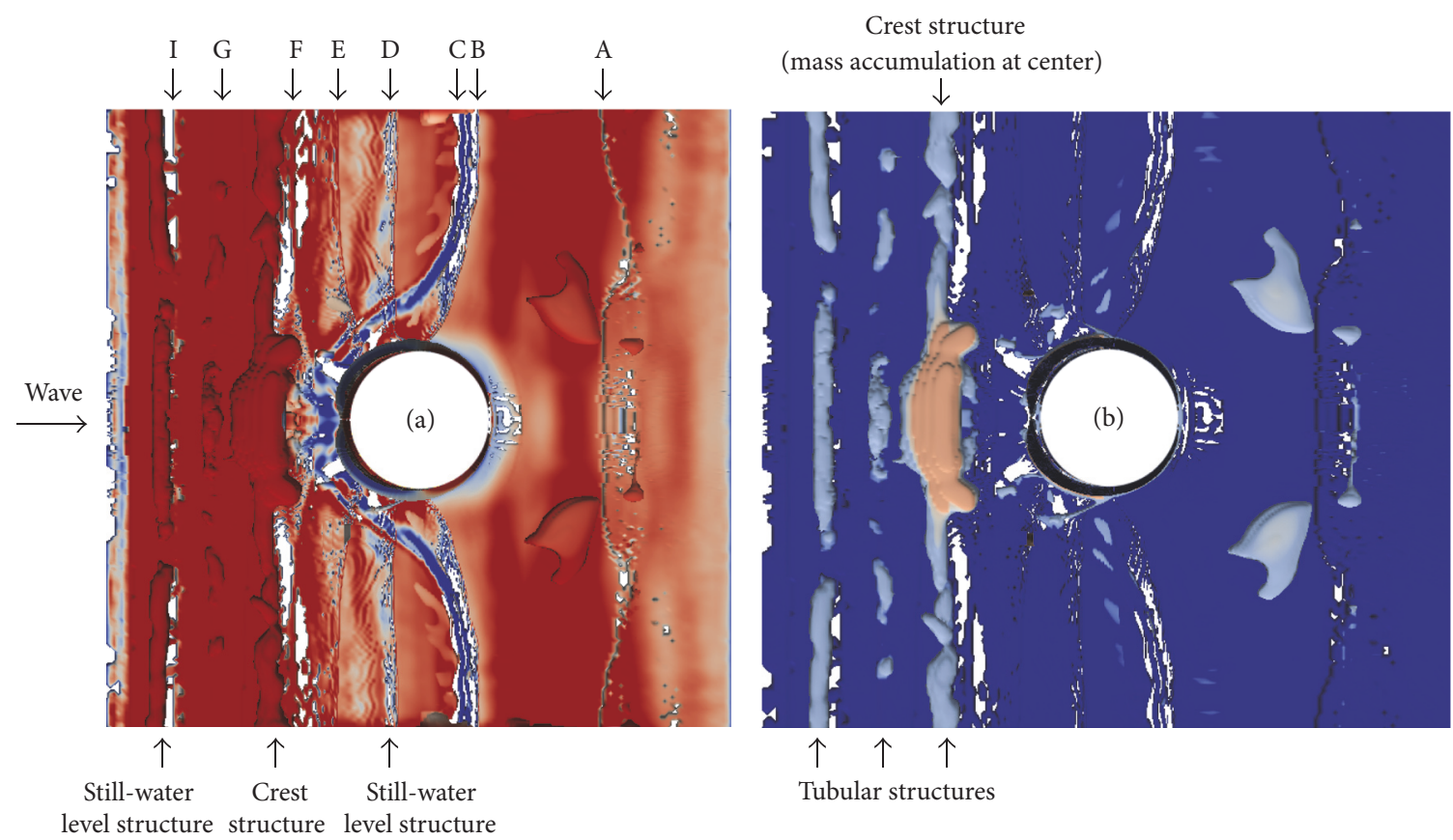

FiguRE 1: Bottom view of structure field $\left(\lambda_{c i}\right.$ criterion) at $t=0$. Structures colored with: (a) spanwise vorticity of wave field; (b) pressure values of wave field.
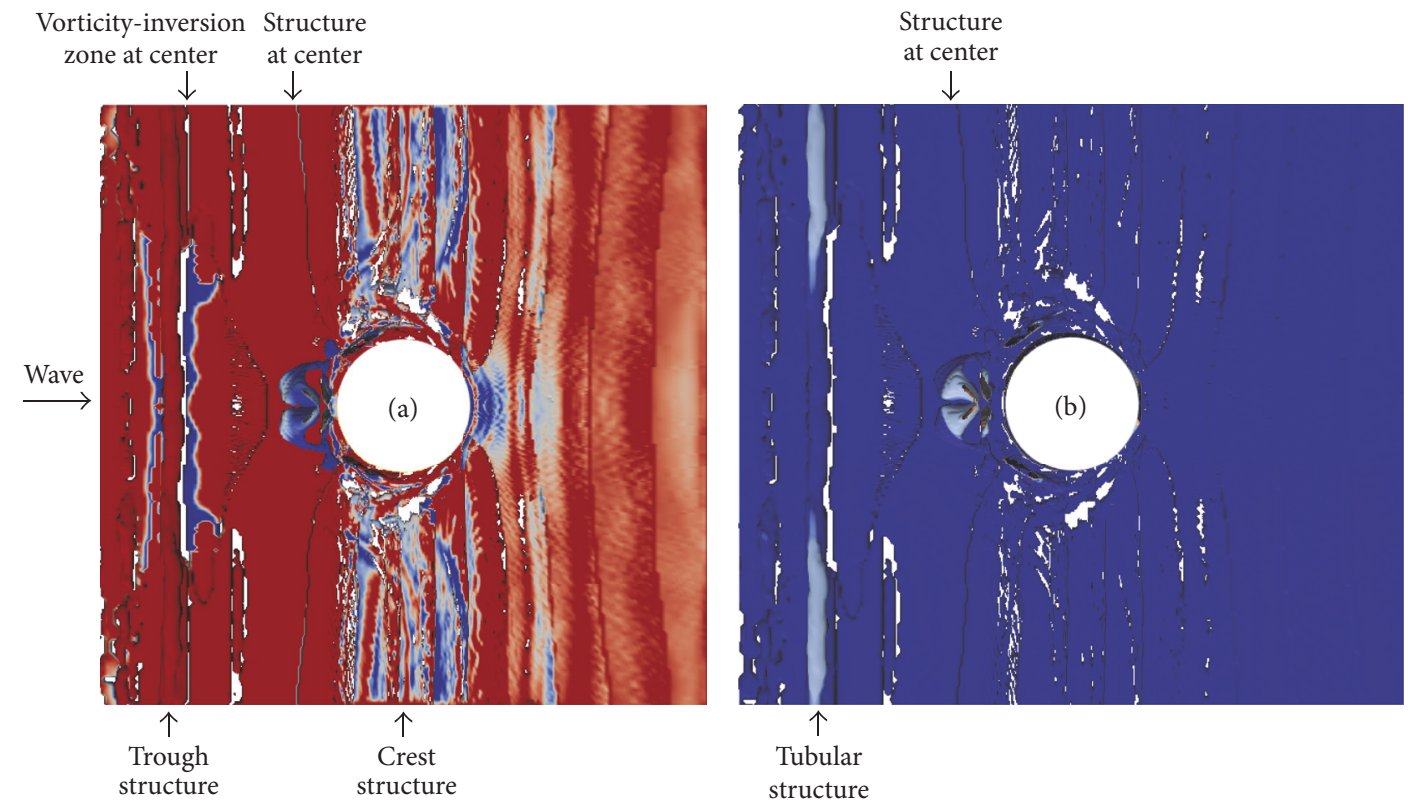

FIGURE 2: Bottom view of structure field $\left(\lambda_{c i}\right.$ criterion) at $t=0.144\left(t=t_{F_{\max }}^{\mathrm{NS}}\right)$. Structures colored with: (a) spanwise vorticity of wave field; (b) pressure values of wave field.

RAM, and 1.8 TB of mass memory (one can refer to [42] for a complete description of the system).

\section{Structures and Modes}

Among the different methods for the identification of the coherent vortical structures in a flow field ([43]), the swirlingstrength criterion (or $\lambda_{c i}$ criterion, see also [27]) has been used (one can refer to [43] for a complete description of the method). In several different flow cases (see [44-46] for more details), this technique has demonstrated to give very satisfactory results. As for the present work, the results of the simulations have been reported by visualizing the flow structures corresponding to a given threshold value of $\lambda_{c i}$ $\left(\left.\lambda_{c i}\right|_{\text {th }}\right)$ [47]. In Figures 1(a) and 2(a) the outer surface of each structure is colored with $\left(\omega_{y}\right.$, the vorticity spanwise component) in a blue-to-red scale, with $\omega_{y}$ varying within a given interval [bluish $\omega_{y}$ (negative) mirrors clockwise local-particle 
TABLE 1: Field instants represented in Figures 1-5.

\begin{tabular}{|c|c|c|c|}
\hline Figures & Instant $(t)$ [ $T$ units $]$ & Instant $(t)[\sqrt{D / g}$ units $]$ & Instant characteristics \\
\hline Figure 1 & 0.000 & 0.000 & Beginning of wave period \\
\hline Figures 2 and 4 & 0.144 & 0.715 & $t=t_{F_{\max }}^{\mathrm{NS}}$ (instant at which $F_{\max }$ is verified $)$ \\
\hline Figures 3 and 5 & 0.257 & 1.276 & $t=t_{R_{\max }}^{\mathrm{NS}}$ (instant at which $R_{\max }$ is verified) \\
\hline
\end{tabular}

rotation, reddish $\omega_{y}$ (positive) mirrors counterclockwise local-particle rotation, greyish areas correspond to quasi-zero $y$-vorticity]. In Figures 1(b) and 2(b), the outer surfaces are further colored with the values of the pressure field, in a dark-to-light scale (near the free surface, bluish areas denote low pressure values and, deeper under the free surface, rosy areas denote higher pressure values; see also Table 1). Then, the figures, in which the coherent structures of the flow are visualized, mirror three types of information: (i) they depict the fluid flow in terms of coherent structures, as extracted with the $\lambda_{c i}$ criterion and shown at given values of $\left.\lambda_{c i}\right|_{\mathrm{th}}$; (ii) the lines that one can eventually detect in the figures represent borders between different structures; (iii) the outer surfaces of the structures are colored with $\omega_{y}$, the spanwise vorticity (or also with the pressure), so that the sign and magnitude of the local-particle rotation can be inferred from the figures themselves (more intense $\omega_{y}$ denotes vortex-line stretching, less intense $\omega_{y}$ denotes shrinking of the vortex-lines).

As for flow modes, the Karhunen-Loève (KL) decomposition has been implemented for the extraction of the most energetic modes of the flow (one can refer to [48-50] for a complete description of the method). Also, this technique has shown to give very interesting results in several different flow cases ([51-55]). For the problem at hand, the computational code has been utilized, as originally developed by Alfonsi and Primavera [56]. Due to limits in our computational resources, a spatial subdomain has been selected within the whole original domain in which the Navier-Stokes computations have been previously executed. The subdomain involves $N_{x}=$ $49, N_{y}=80, N_{z}=10$ points $\left(N_{\text {tot }}=39200\right)$, and is localized right upstream from the cylinder (Figures 4 and 5).

As a result of the decomposition, a total of $\left(3 \times N_{x} \times N_{y} \times\right.$ $\left.N_{z}=117600\right)$ three-component eigenfunctions with related eigenvalues, have been computed. It resulted, as expected, in that the first three eigenfunctions alone exhibit a cumulative energy content of more than $99.8 \%$ of the total kinetic energy of the flow field. Thus, a reduced three-dimensional, timedependent velocity field in the subdomain has been reconstructed by taking into account only the three most energetic eigenfunctions. As for visualizations, the reduced velocity fields have been reported by visualizing surfaces of constant wave-field velocity components at given threshold values.

\section{Results}

As for forces and run-up values, Figure 10 in [21] shows the maxima values of the force (adimensional) on the cylindrical body as obtained in the calculations, in comparison with linear theory, numerical solution of the Euler equations, and experimental data. Both linear-theory and Euler-equation results slightly underestimate the force with respect to the results given by both the Navier-Stokes calculations and the experiments (at similar values of $k r_{0}$ ). It is to be noted here that the general good agreement between potential-theory and Euler-equation results shows that, in the inviscid-fluid framework, both potential theory and mass- and momentum equations give, in practice, the same results. It is then confirmed that all differences that can be found between inviscid and viscous fluid results, are due to the effects of the shear-stresses (generated by the viscosity).

In an analogous manner, Figure 11 in [21] shows the maxima values of the run-up (adimensional) on the cylindrical body as obtained in the calculations, again in comparison with linear theory, numerical solution of the Euler equations, and experimental data. The circumstance that linear theory (and also Euler-equation results) strongly underestimates the viscous-fluid wave run-up is here confirmed by both NavierStokes simulations and experimental data.

As for wave-flow structures and energy modes (see also Table 1), Figures 1-3 show bottom views of the flow fields through a sequence of instants, where "(a)" images show the $\lambda_{c i}$ derived flow structures (colored with $\omega_{y}$ as previously outlined) and "(b)" images show the same structures colored with the wave-field pressure values. From Figure 1(a) $(t=0)$, it appears that the free-surface tracks $\mathrm{B}$ and $\mathrm{C}$ are the borders of a structure that is noticeably contaminated by negative spanwise vorticity under the free-surface level, while tracks F, $\mathrm{G}$, and I mirror the presence of remarkably developed underwater tubular structures with a high vorticity content. The wave-crest structure (Figures $1(\mathrm{a})$ and $1(\mathrm{~b})$ ) is characterized by a remarkable phenomenon of mass accumulation right in front of the cylinder.

From Figure 2(a) $\left(t=0.144, t=t_{F_{\max }}^{\mathrm{NS}}\right)$ and right upstream from the cylinder, it is possible to observe a spanwise tubular structure as part of a complex wave-trough structure, the latter including the so-called vorticity-inversion zone (one can refer to [21] for a complete description of this phenomenon). Figure 2(b) also shows that the peripheral zone of the latter tubular structure lies more in depth with respect to its central part. Figures 2(a) and 2(b) further show, right upstream from the cylinder, a central structure under the free-surface level, as characterized by negative vorticity (Figure 2(a)) and remarkable depth (Figure 2(b)).

Figure $3\left(t=0.257, t=t_{R_{\max }}^{\mathrm{NS}}\right)$ shows the flow fields at the instant in which the wave exhibits its maximum runup on the cylinder. Also, here a complex tubular structure can be recognized upstream from the cylinder, the latter showing at center a massive substructure. Note that this substructure is characterized by less-intense spanwise vorticity than other portions of the same structure (Figure 3(a)). 

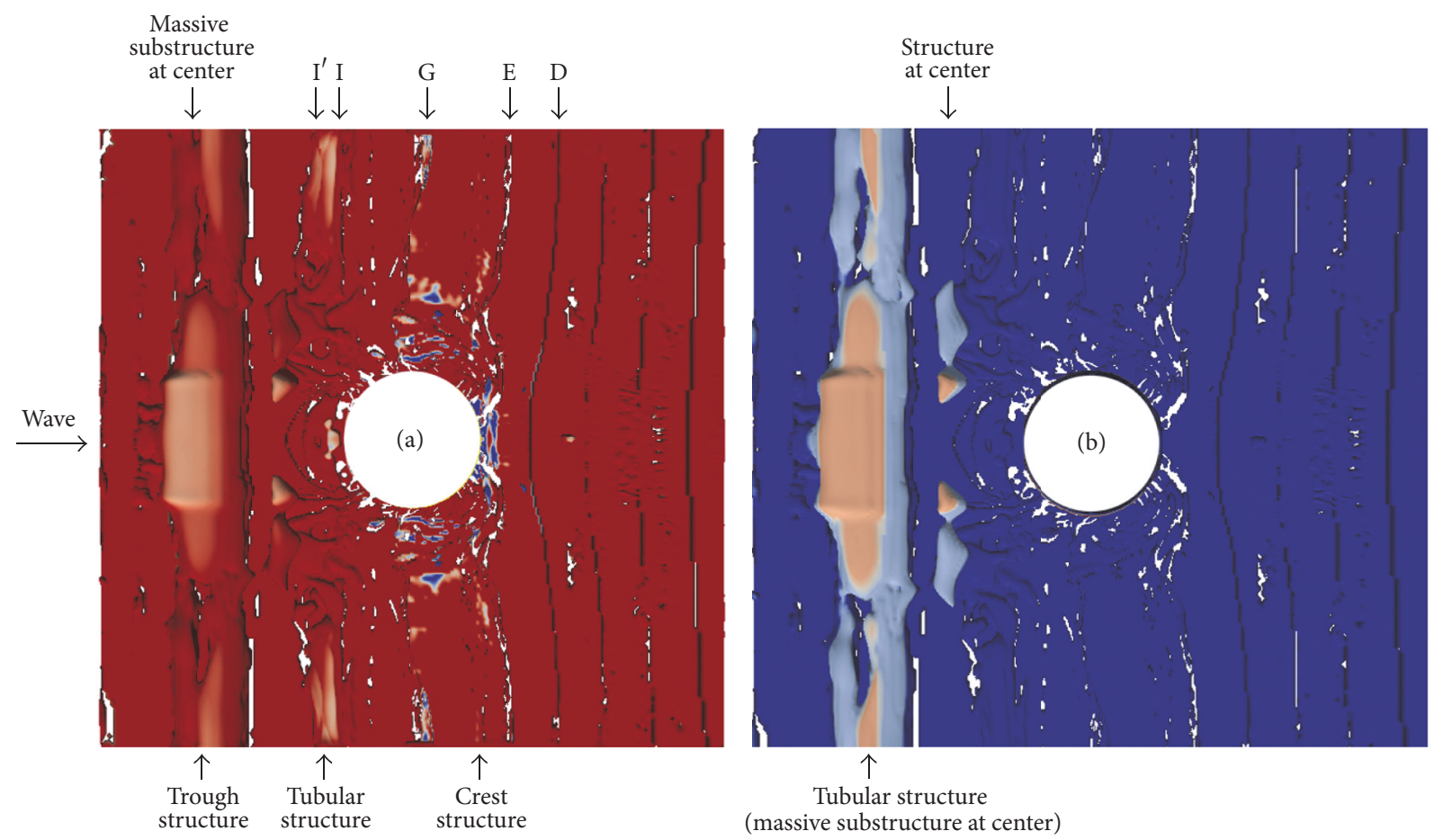

FIGURE 3: Bottom view of structure field $\left(\lambda_{c i}\right.$ criterion) at $t=0.257\left(t=t_{R_{\max }}^{\mathrm{NS}}\right)$. Structures colored with: (a) spanwise vorticity of wave field; (b) pressure values of wave field.

From Figure 3(b), it can be noted that the substructure extends noticeably in depth under the free surface, differently from what is shown in Figure 2(b) (previous instant), where, upstream from the cylinder, the central part of the trough structure lies practically at the free-surface level. Figures $3(\mathrm{a})$ and 3(b) also show the central structure, as now characterized by positive vorticity and remarkable depth (the structure has broken up in two parts).

Figures 4 and 5 show bottom-view visualizations at $t=$ $t_{F_{\max }}^{\mathrm{NS}}$ and $t=t_{R_{\max }}^{\mathrm{NS}}$ of the structure field in terms of isosurfaces of the KL-mode-reconstructed velocity field, where "(a)," "(b)," and "(c)" images are related to the velocity components $u, v$, and $w$, respectively. In these figures, relatively large threshold values of $u, v, w$ have been selected in an attempt of unveiling the most energetic structures of the field. In Figure 4(a), isosurfaces of the reduced-field $x$-velocity component are shown from underwater. It can be noticed that the high-kinetic-energy flow structures are organized in smalland larger flow scales, where, in particular, Zone A is a smallscale area located in front of the cylinder, Zone B is a smallscale area adjacent to the former, and Zone $\mathrm{C}$ is a large-scale peripheral area.

Borders can be visually identified among these zones, as depicted in Figure 4( $\left.\mathrm{a}^{\prime}\right)$.

In going from $t_{F_{\max }}^{\mathrm{NS}}$ to $t_{R_{\max }}^{\mathrm{NS}}$ (Figure 5(a)), one can notice that Zone A has noticeably grown in extent, while the border of Zone $\mathrm{C}$ is slightly more flattened with respect to the previous instant. Interestingly, the density of the high-energy flow structures, especially in Zone A, is now much higher than before (the $u$ - threshold value is the same) and a similar phenomenon also occurs as related to the $z$-velocity component $(w)$. At $t_{F_{\max }}^{\mathrm{NS}}$ (Figure 5(c)), the field of the iso$w$ structures is characterized by the presence of a quasitriangular small-scale zone right in front of the cylinder (arrows 6) and of another zone, upstream from the former (arrows 5). In going from $t_{F_{\max }}^{\mathrm{NS}}$ to $t_{R_{\max }}^{\mathrm{NS}}$, both zones have grown in extent (Figure $5(\mathrm{c})$ ), and the density of the high-energy flow structures is now much higher than it was at the previous instant (the $w$ - threshold is the same). Thus, in front of the cylinder, both the $u$ - and the $w$ - kinetic-energy contents are remarkably higher at $t_{R_{\max }}^{\mathrm{NS}}$ than at $t_{F_{\max }}^{\mathrm{NS}}$.

\section{Conclusions}

The results obtained in this work actually suggest an explanation of the main nonintuitive character of the process of interaction between a wave and a surface-piercing cylinder, namely, the fact that the wave field exerts its maximum force and exhibits its maximum run-up at different instants $\left[\left(t_{F_{\max }}\right),\left(t_{R_{\max }}\right)\right]$, the former before the latter.

The results show that the flow field, at the instant in which the wave exerts its maximum force, is characterized (i) by the most complex configuration of underwater structures, mainly at the upstream cylinder wall, and, (ii) by the presence of an oncoming wavefront toward the cylinder whose strength actually causes the so-called vorticity-inversion phenomenon (the latter extensively described in [21]). These two simultaneous flow phenomena actually determine the maximum inline force on the cylinder. Furthermore, Figure 5 tells us that the instant $\left(t_{R_{\max }}\right)$ at which the wave field exhibits 

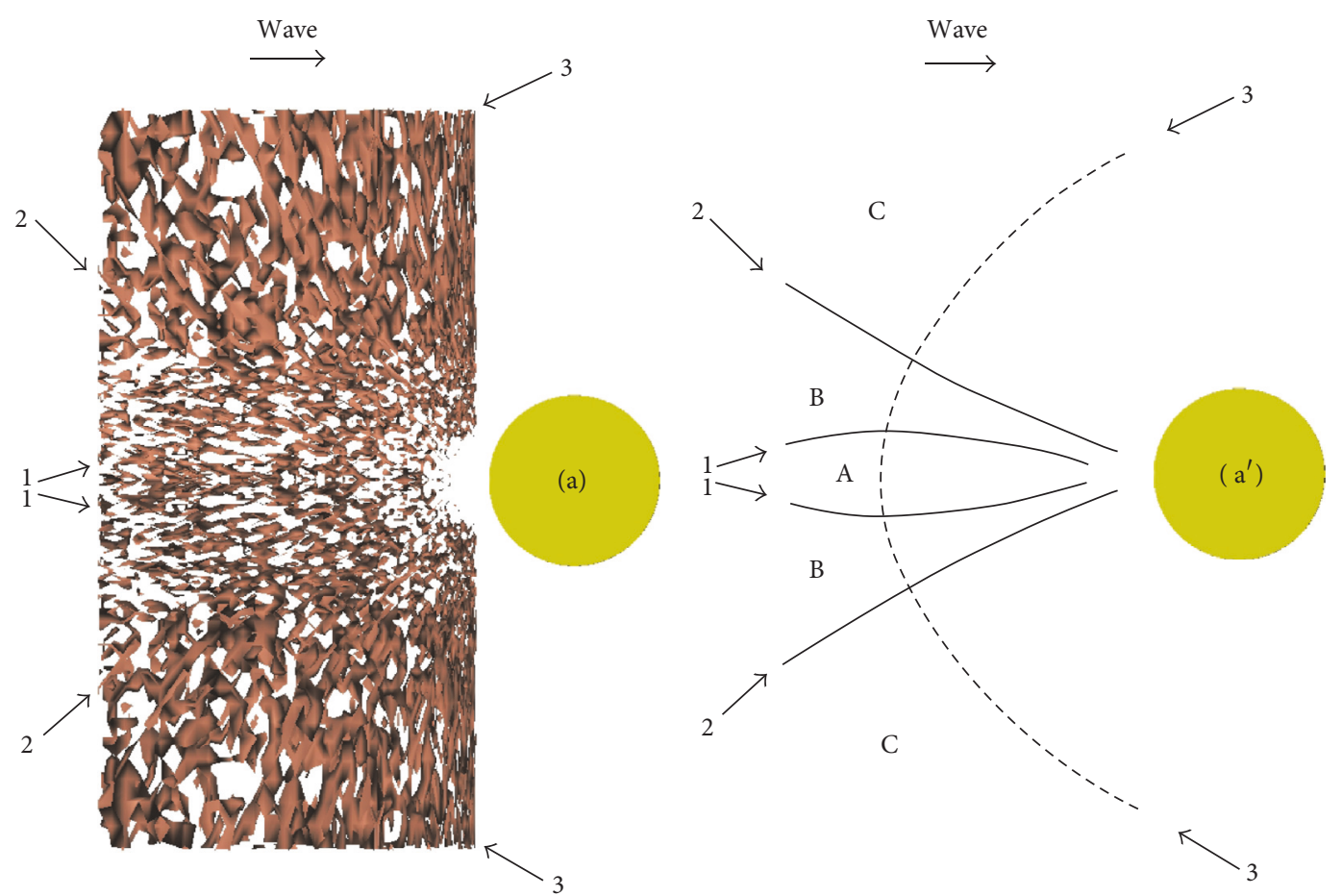

A: iso- $u$ small-scale zone

$\mathrm{B}$ : iso- $u$ small-scale zones

$\mathrm{C}$ : iso- $u$ large-scale zones
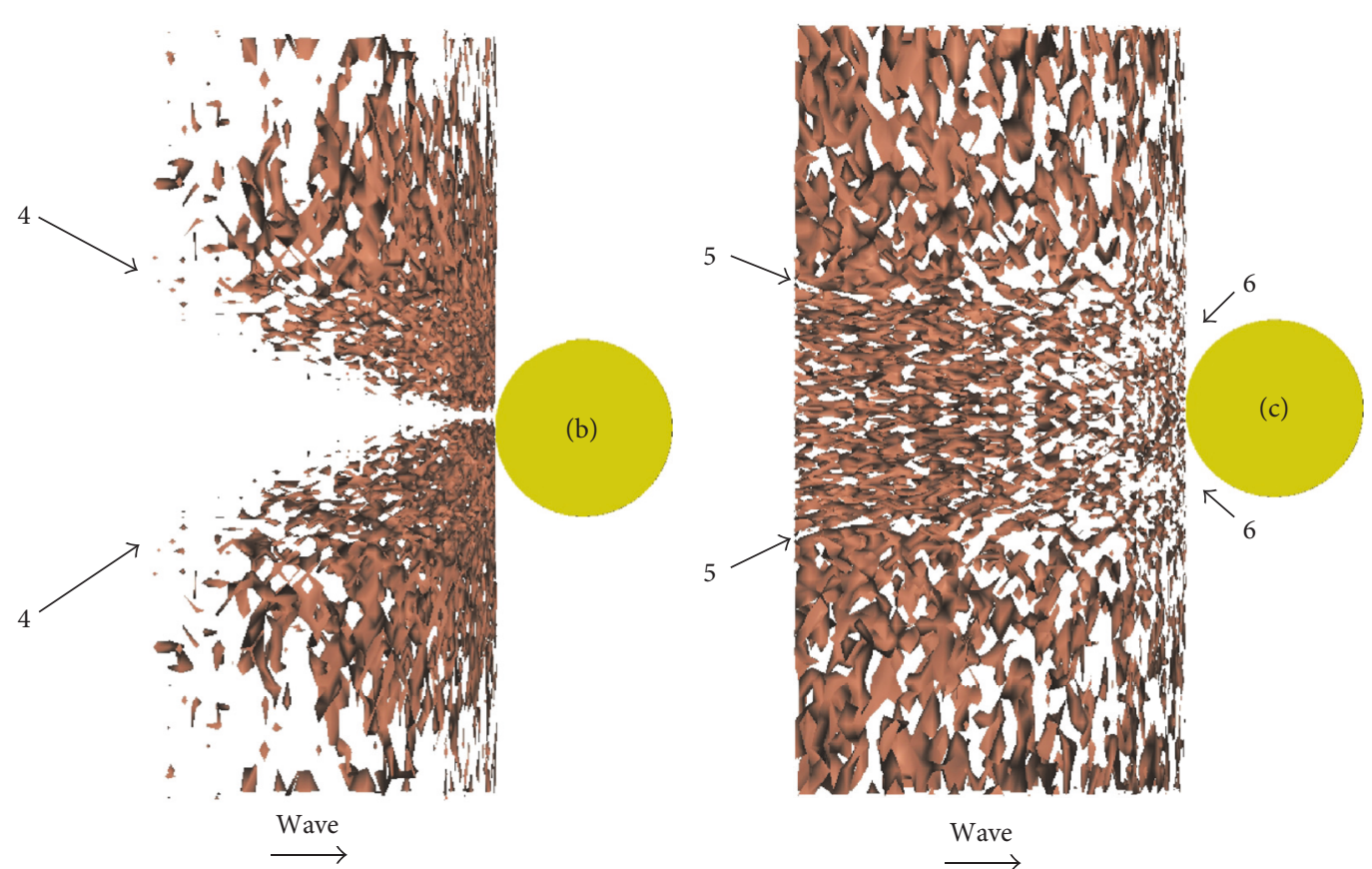

FIGURE 4: Bottom view of constant-velocity surfaces from KL-mode reconstruction at $t=t_{F_{\max }}^{\mathrm{NS}}:(\mathrm{a}) x$-component $(u)$; (a') scheme of $u$-modes spatial organization; (b) $y$-component $(v)$; (c) $z$-component $(w)$. 

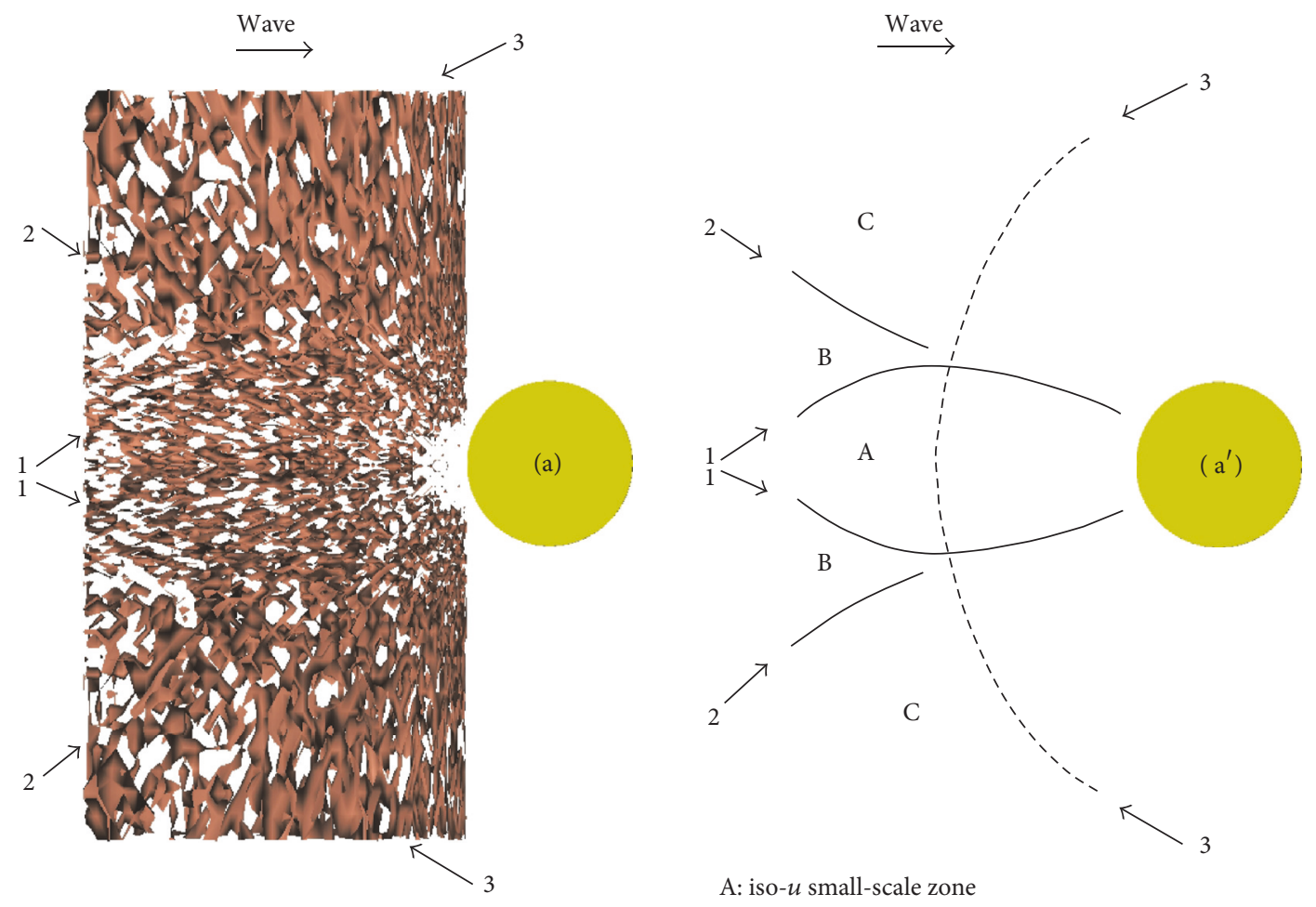

A: iso- $u$ small-scale zone

$\mathrm{B}$ : iso- $u$ small-scale zones

C: iso- $u$ large-scale zones
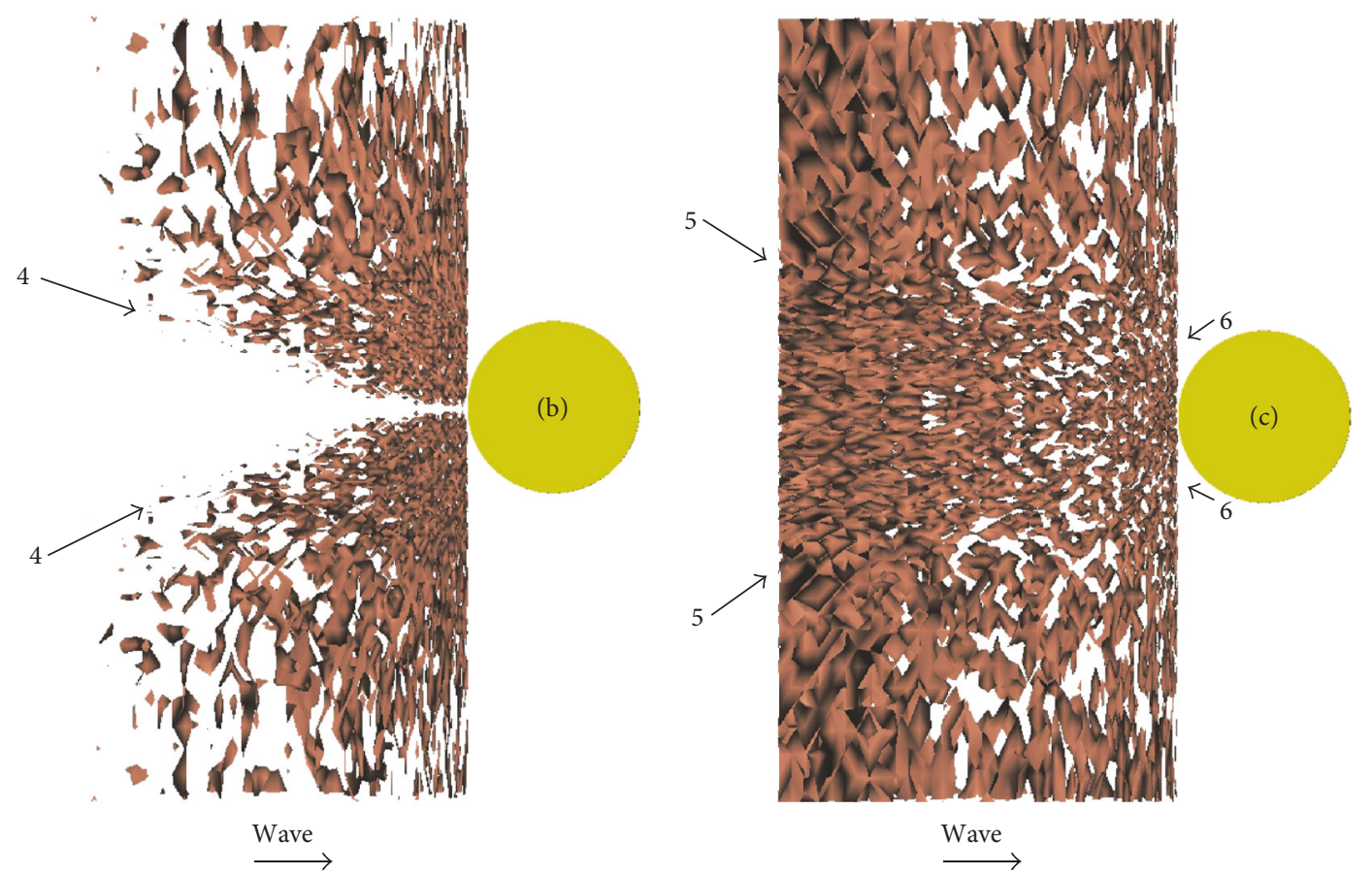

FIGURE 5: Bottom view of constant-velocity surfaces from KL-mode reconstruction at $t=t_{R_{\max }}^{\mathrm{NS}}:(\mathrm{a}) x$-component $(u)$; (a') scheme of $u$-modes spatial organization; (b) $y$-component $(v)$; (c) $z$-component $(w)$. 
its maximum run-up is characterized by the maximum density of high-kinetic-energy flow structures in the proximity of the obstacle. This high-energy content of the wave flow actually determines the aforementioned maximum run-up.

\section{Nomenclature}

\section{Roman Symbols (Upper Case)}

\begin{tabular}{|c|c|}
\hline$D:$ & Cylinder diameter \\
\hline$F_{\max }:$ & Maximum force on cylinder \\
\hline$F_{\max }^{\mathrm{ND}}:$ & $\begin{array}{l}\text { Maximum force on cylinder } \\
\text { (nondimensional) }\end{array}$ \\
\hline$H:$ & Wave height \\
\hline KC: & Keulegan-Carpenter number \\
\hline$L:$ & Wave length \\
\hline$L_{x}, L_{y}, L_{z}:$ & Dimensions of computing domain along \\
\hline$N_{x}, N_{y}, N_{z}:$ & $\begin{array}{l}x, y, z \\
\text { Number of grid points in the computing } \\
\text { domain along } x, y, z\end{array}$ \\
\hline$N_{\text {tot }}:$ & $\begin{array}{l}\text { Total number of grid points in the } \\
\text { computing domain }\end{array}$ \\
\hline$R_{\max }:$ & Maximum run-up at cylinder surface \\
\hline$R_{\max }^{\mathrm{ND}}$ & $\begin{array}{l}\text { Maximum run-up at cylinder surface } \\
\text { (nondimensional) }\end{array}$ \\
\hline Re: & Wave-field Reynolds number \\
\hline $\operatorname{Re}_{\mathrm{NS}}$ : & $\begin{array}{l}\text { Reynolds number resulting from } \\
\text { adimensionalization of NS equations }\end{array}$ \\
\hline$T:$ & Wave period. \\
\hline
\end{tabular}

\section{Roman Symbols (Lower Case)}

$\begin{array}{ll}a: & \text { Wave amplitude } \\ d: & \text { Still-water level } \\ g: & \text { Acceleration due to gravity } \\ & \left(g=9.807 \mathrm{~m} / \mathrm{s}^{2}\right) \\ p: & \text { Pressure }\end{array}$

$r, \theta, z: \quad$ Cylindrical coordinate system

$r_{0}: \quad$ Cylinder radius

$t: \quad$ Time coordinate

$t_{F_{\max }}^{\mathrm{NS}}: \quad$ Time at which the

Navier-Stokes-equations-derived $F_{\max }$ is verified

$t_{R_{\max }}^{\mathrm{NS}}: \quad$ Time at which the Navier-Stokes-equations-derived $R_{\max }$ is verified

$u_{i}(u, v, w)$ : Velocity components along $x, y, z$

$u_{\text {max }}: \quad$ Maximum value of $u$

$x_{i}(x, y, z)$ : Cartesian coordinates ( $x$ is wave direction, $z$ is vertical direction).

\section{Greek Symbols (Upper Case)}

$\Delta t: \quad$ Time resolution of calculations

$\Delta x, \Delta y, \Delta z$ : Space resolution of calculations along $x, y$, $z$.

Greek Symbols (Lower Case)

$\lambda_{c i}$ : Imaginary part of complex eigenvalue $\left(\lambda_{c i}\right)_{\mathrm{th}}:$ Threshold value of swirling strength

$\delta_{i j}: \quad$ Kronecker delta

$\bar{\varepsilon}: \quad$ Average rate of dissipation of kinetic energy per unit mass

$\eta$ : Kolmogorov length scale

$\mu$ : $\quad$ Fluid dynamic viscosity

$v$ : $\quad$ Fluid kinematic viscosity

$\tau_{\eta}: \quad$ Kolmogorov time scale

$\rho: \quad$ Fluid density

$\omega_{i}$ : Vorticity vector.

\section{Acronyms}

DNS: Direct Numerical Simulation (technique)

KL: Karhunen-Loève decomposition (technique)

RANS: Reynolds Averaged Navier-Stokes (equations)

RNG: Renormalization Group (theory).

\section{Conflicts of Interest}

The authors declare that there are no conflicts of interest regarding the publication of this paper.

\section{References}

[1] R. C. MacCamy and R. A. Fuchs, "Wave forces on piles: a diffraction theory," Technical Memorandum 69, US Army Corp of Engineers, Beach Erosion Board, Washington, DC, USA, 1954.

[2] A. M. Mansour, A. N. Williams, and K. H. Wang, "The diffraction of linear waves by a uniform vertical cylinder with cosinetype radial perturbations," Ocean Engineering, vol. 29, no. 3, pp. 239-259, 2001.

[3] T. Sarpkaya and M. Isaacson, "Mechanics of wave forces in offshore structures," Van Nostrand Reinhold, 1981.

[4] P. D. Roy and S. Ghosh, "Wave force on vertically submerged circular thin plate in shallow water," Ocean Engineering, vol. 33, no. 14-15, pp. 1935-1953, 2006.

[5] N. H. Kim, M. S. Park, and S. B. Yang, "Wave force analysis of the vertical circular cylinder by boundary element method," KSCE Journal of Civil Engineering, vol. 11, no. 1, pp. 31-35, 2007.

[6] B. Helstrom and L. Rundgren, Model Tests on Oland Sodva Grund Light-House, vol. 39, Royal Institute of Technology Institute of Hydraulics Bulletin, Stockolm, Sweden, 1954.

[7] A. D. K. Laird, "A model study of wave action on a cylindrical island," Eos, Transactions, American Geophysical Union, vol. 36, no. 2, pp. 279-285, 1955.

[8] R. Bonnefille and P. Germain, "Wave action on isolated vertical cylinders of large dimension," in Proceedings of the IARH World Congress, pp. 311-318, 1963.

[9] M. Isaacson, "Wave runup around large circular cylinder," ASCE Journal of Waterways and Harbors, Coastal Engineering Division, vol. 104, pp. 69-79, 1978.

[10] C. J. Galvin and R. J. Hallermeier, "Wave Runup on Vertical Cylinders," in Proceedings of the 13th International Conference on Coastal Engineering, pp. 1955-1974, 1972.

[11] R. J. Hallermeier, "Nonlinear flow of wave crests past a thin pile," ASCE Journal of Waterways and Harbors, Coastal Engineering Division, vol. 102, pp. 365-377, 1976. 
[12] J. P. Haney and J. B. Herbich, "Wave flow around thin piles and pile groups," Journal of Hydraulic Research, vol. 20, no. 1, pp. 114,1982 .

[13] S. Chakrabarti and W. A. Tam, "Wave height distribution around vertical cylinder," ASCE Journal of Waterways and Harbors, Coastal Engineering Division, vol. 101, pp. 225-230, 1975.

[14] J. Niedzwecki and A. Duggal, "Wave run-up and wave forces on a truncated cylinder," in Proceedings of 22nd Offshore Technology Conference, pp. 593-600, 1990.

[15] J. M. Niedzwecki and A. S. Duggal, "Wave runup and forces on cylinders in regular and random waves," Journal of Waterway, Port, Coastal, and Ocean Engineering, vol. 118, no. 6, pp. 615634, 1992.

[16] J. M. Niedzwecki and J. R. Huston, "Wave interaction with tension leg platforms," Ocean Engineering, vol. 19, no. 1, pp. 2137, 1992.

[17] A. J. Martin, W. J. Easson, and T. Bruce, "Runup on columns in steep, deep water regular waves," Journal of Waterway, Port, Coastal, and Ocean Engineering, vol. 127, no. 1, pp. 26-32, 2001.

[18] H. Mase, K. Kosho, and S. Nagahashi, "Wave runup of random waves on a small circular pier on sloping seabed," Journal of Waterway, Port, Coastal, and Ocean Engineering, vol. 127, no. 4, pp. 192-199, 2001.

[19] M. T. Morris-Thomas and K. P. Thiagarajan, “The run-up on a cylinder in progressive surface gravity waves: Harmonic components," Applied Ocean Research, vol. 26, no. 3-4, pp. 98-113, 2004.

[20] L. De Vos, P. Frigaard, and J. De Rouck, "Wave run-up on cylindrical and cone shaped foundations for offshore wind turbines," Coastal Engineering Journal, vol. 54, no. 1, pp. 17-29, 2007.

[21] G. Alfonsi, "Numerical simulations of wave-induced flow fields around large-diameter surface piercing vertical circular cylinder," Computation, vol. 3, pp. 386-426, 2015.

[22] B. H. Choi, D. C. Kim, E. Pelinovsky, and S. B. Woo, "Threedimensional simulation of tsunami run-up around conical island," Coastal Engineering Journal, vol. 54, no. 8, pp. 618-629, 2007.

[23] G. Alfonsi, "Reynolds-averaged Navier-Stokes equations for turbulence modeling," Applied Mechanics Reviews, vol. 62, no. 4, pp. 1-20, 2009.

[24] G. Alfonsi, A. Lauria, and L. Primavera, "Structures of a viscouswave flow around a large-diameter circular cylinder," Journal of Flow Visualization and Image Processing, vol. 19, no. 4, pp. 323354, 2012.

[25] G. Alfonsi, A. Lauria, and L. Primavera, "Flow structures around a large-diameter circular cylinder," Journal of Flow Visualization and Image Processing, vol. 19, no. 1, pp. 15-35, 2012.

[26] G. Alfonsi, "On Direct Numerical Simulation of Turbulent Flows," Applied Mechanics Reviews, vol. 64, article 020802, pp. 1-33, 2011.

[27] J. Zhou, R. J. Adrian, S. Balachandar, and T. . Kendall, "Mechanisms for generating coherent packets of hairpin vortices in channel flow," Journal of Fluid Mechanics, vol. 387, pp. 353-396, 1999.

[28] Flow Science, Inc. Flow-3D User Manual. Santa Fe, NM, 2004.

[29] C. W. Hirt and B. D. Nichols, "Volume of fluid (VOF) method for the dynamics of free boundaries," Journal of Computational Physics, vol. 39, no. 1, pp. 201-225, 1981.
[30] S. Kocaman, G. Seckin, and K. S. Erduran, "3D model for prediction of flow profiles around bridges," Journal of Hydraulic Research, vol. 48, no. 4, pp. 521-525, 2010.

[31] S. M. Cho, "Foundation design of the Incheon bridge," Geotechnical Engineering Journal, vol. 41, pp. 1-16, 2010.

[32] J. Jin and B. Meng, "Computation of wave loads on the superstructures of coastal highway bridges," Ocean Engineering, vol. 38, no. 17-18, pp. 2185-2200, 2011.

[33] C. Kim, J. Kim, and J. Kang, "Analysis of the cause for the collapse of a temporary bridge using numerical simulation," Engineering Journal, vol. 5, no. 12, pp. 997-1005, 2013.

[34] M. Kayser and M. Gabr, "Assessment of scour on bridge foundations by means of in situ erosion evaluation probe," Transportation Research Record, no. 2335, pp. 72-78, 2013.

[35] G. Alfonsi, G. Passoni, L. Pancaldo, and D. Zampaglione, "A spectral-finite difference solution of the Navier-Stokes equations in three dimensions," International Journal for Numerical Methods in Fluids, vol. 28, no. 1, pp. 129-142, 1998.

[36] G. Passoni, G. Alfonsi, G. Tula, and U. Cardu, "A wavenumber parallel computational code for the numerical integration of the navier-stokes equations," Parallell Computing, vol. 25, no. 5, pp. 593-611, 1999.

[37] G. Passoni, P. Cremonesi, and G. Alfonsi, "Analysis and implementation of a parallelization strategy on a navier-stokes solver for shear flow simulations," Parallel Computing. Systems \& Applications, vol. 27, no. 13, pp. 1665-1685, 2001.

[38] G. Passoni, G. Alfonsi, and M. Galbiati, "Analysis of hybrid algorithms for the Navier-Stokes equations with respect to hydrodynamic stability theory," International Journal for Numerical Methods in Fluids, vol. 38, no. 11, pp. 1069-1089, 2002.

[39] G. Alfonsi and L. Primavera, "Direct numerical simulation of turbulent channel flow with mixed spectral-finite difference technique," Journal of Flow Visualization and Image Processing, vol. 14, no. 2, pp. 225-243, 2007.

[40] H. P. Bakewell Jr. and J. L. Lumley, "Viscous sublayer and adjacent wall region in turbulent pipe flow," Physics of Fluids, vol. 10, no. 9, pp. 1880-1889, 1967.

[41] P. W. Bearman, M. J. Downie, J. M. R. Graham, and E. D. Obasaju, "Forces on cylinders in viscous oscillatory flow at low Keulegan-Carpenter numbers," Journal of Fluid Mechanics, vol. 154, no. 7, pp. 337-356, 1985.

[42] G. Alfonsi, S. A. Ciliberti, M. Mancini, and L. Primavera, "GPGPU implementation of mixed spectral-finite difference computational code for the numerical integration of the three-dimensional time-dependent incompressible NavierStokes equations," Computers \& Fluids, vol. 102, pp. 237-249, 2014.

[43] L. Primavera and G. Alfonsi, "On identification of vortical structures in turbulent shear flow," Journal of Flow Visualization and Image Processing, vol. 15, no. 3, pp. 201-216, 2008.

[44] G. Alfonsi and L. Primavera, "Temporal evolution of vortical structures in the wall region of turbulent channel flow," Flow, Turbulence and Combustion, vol. 83, no. 1, pp. 61-79, 2009.

[45] G. Alfonsi, A. Lauria, and L. Primavera, "A study of vortical structures past the lower portion of the ahmed car model," Journal of Flow Visualization and Image Processing, vol. 19, no. 1, pp. 81-95, 2012.

[46] G. Alfonsi, S. A. Ciliberti, M. Mancini, and L. Primavera, "Turbulent events in a wall-bounded turbulent flow," Journal of Flow Visualization and Image Processing, vol. 19, no. 2, pp. 139-160, 2012. 
[47] G. Alfonsi and L. Primavera, "Determination of the threshold value of the quantity chosen for vortex representation in turbulent flow," Journal of Flow Visualization and Image Processing, vol. 16, no. 1, pp. 41-49, 2009.

[48] J. L. Lumley, Stochastic tools in turbulence, Academic Press, 1971.

[49] L. Sirovich, "Turbulence and the dynamics of coherent structures. Part I: coherent structures. Part II: symmetries and transformations. Part III: dynamics and scaling," Quarterly of Applied Mathematics, vol. 45, no. 3, pp. 561-590, 1987.

[50] G. Alfonsi, "Coherent structures of turbulence: Methods of eduction and results," Applied Mechanics Reviews, vol. 59, no. 1-6, pp. 307-323, 2006.

[51] G. Alfonsi and L. Primavera, "Coherent structure dynamics in turbulent channel flow," Journal of Flow Visualization and Image Processing, vol. 9, no. 1, pp. 89-98, 2002.

[52] G. Alfonsi and L. Primavera, "The structure of turbulent boundary layers in the wall region of plane channel flow," Proceedings of the Royal Society A Mathematical, Physical and Engineering Sciences, vol. 463, no. 2078, pp. 593-612, 2007.

[53] G. Alfonsi, C. Restanto, and L. Primavera, "Coherent structures of the flow around a surface-mounted cubic obstacle in turbulent channel flow," Journal of Wind Engineering \& Industrial Aerodynamics, vol. 91, no. 4, pp. 495-511, 2003.

[54] V. Carbone, F. Lepreti, L. Primavera et al., "An analysis of the vertical photospheric velocity field as observed by Themis," Astronomy \& Astrophysics, vol. 381, no. 1, pp. 265-270, 2002.

[55] A. Vecchio, V. Carbone, F. Lepreti et al., "Proper orthogonal decomposition of solar photospheric motions," Physical Review Letters, vol. 95, no. 6, Article ID 061102, 2005.

[56] G. Alfonsi and L. Primavera, "A parallel computational code for the proper orthogonal decomposition of turbulent flows," Journal of Flow Visualization and Image Processing, vol. 14, no. 3, pp. 267-286, 2007. 


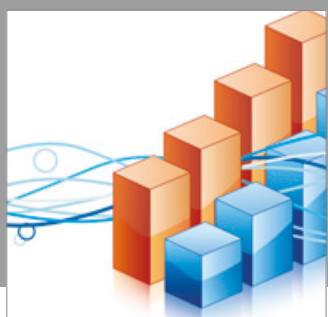

Advances in

Operations Research

vatersals

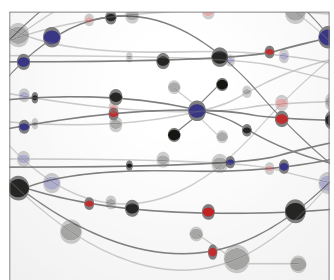

\section{The Scientific} World Journal
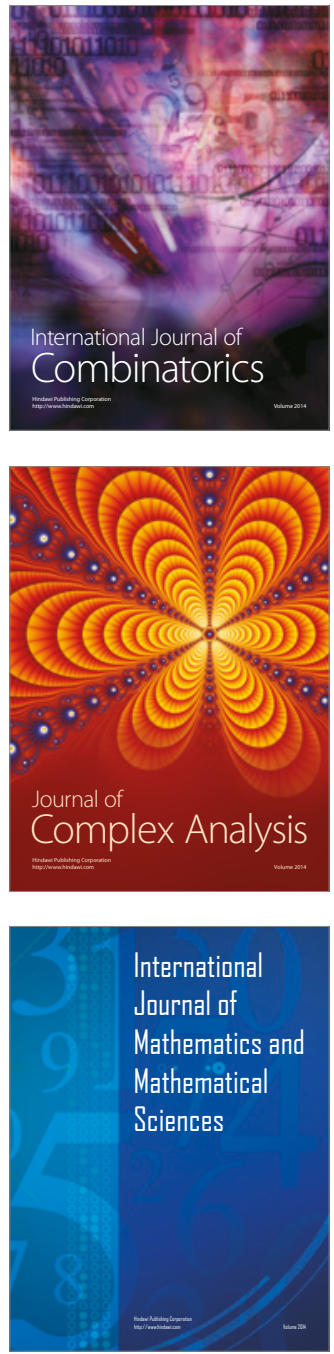
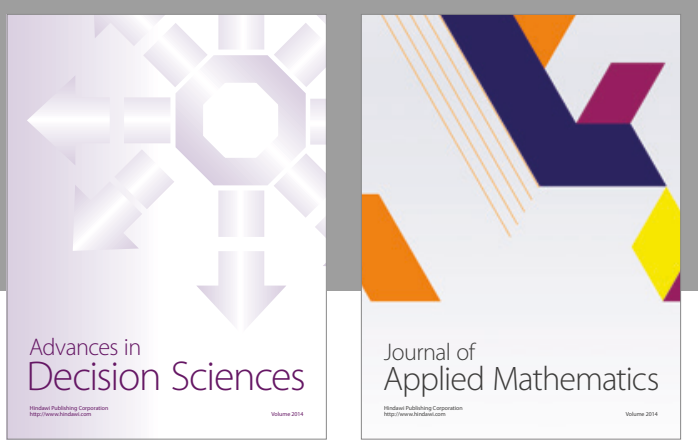

Algebra

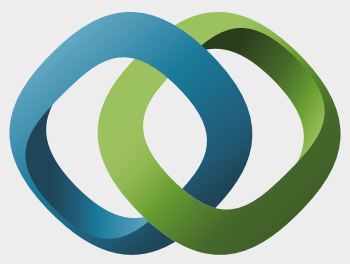

\section{Hindawi}

Submit your manuscripts at

https://www.hindawi.com
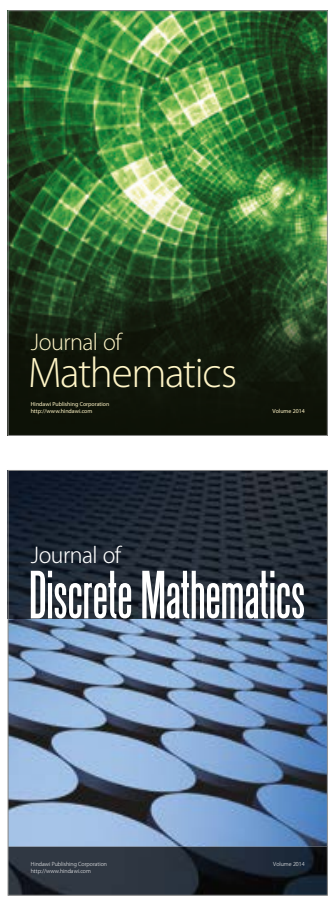

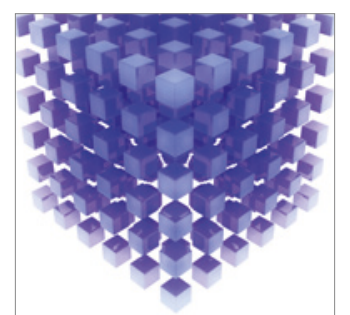

Mathematical Problems in Engineering
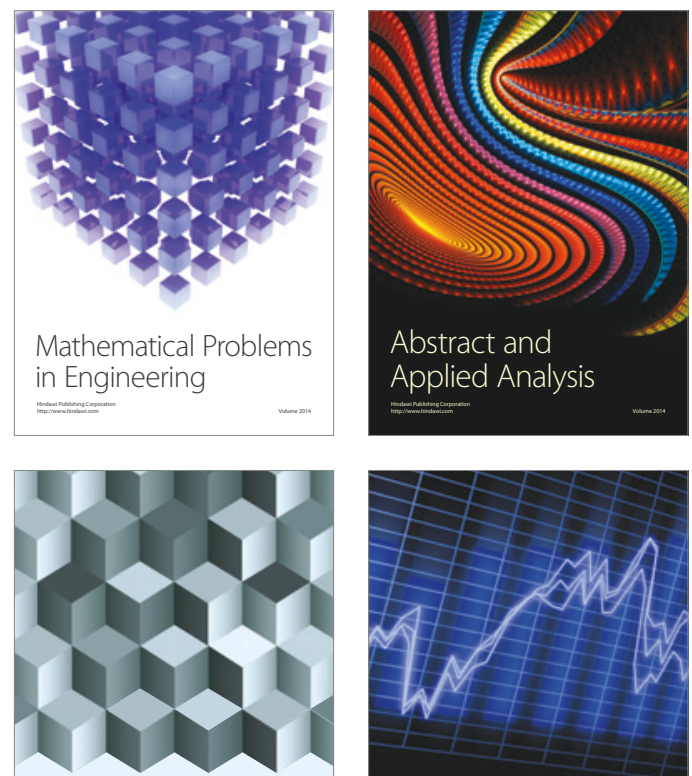

Journal of

Function Spaces

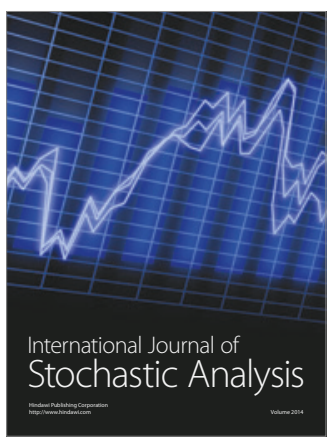

Probability and Statistics
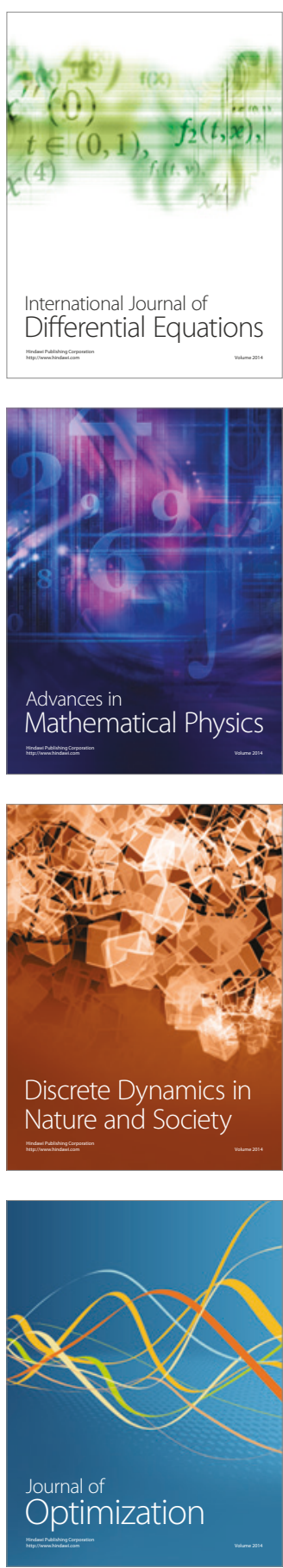